\title{
Beam characterization of FLASH from beam profile measurement by intensity transport equation and reconstruction of the Wigner distribution function
}

Bernd Schäfer, Tobias Mey, Klaus Mann, Barbara Keitel, Svea Kreis, et al.

Bernd Schäfer, Tobias Mey, Klaus Mann, Barbara Keitel, Svea Kreis, Marion Kuhlmann, Elke Plönjes, Kai Tiedtke, "Beam characterization of FLASH from beam profile measurement by intensity transport equation and reconstruction of the Wigner distribution function," Proc. SPIE 8778, Advances in X-ray Free-Electron Lasers II: Instrumentation, 877810 (3 May 2013); doi: 10.1117/12.2016975

SPIE. Event: SPIE Optics + Optoelectronics, 2013, Prague, Czech Republic 


\title{
Beam characterization of FLASH from beam profile measurement by intensity transport equation and reconstruction of the Wigner distribution function
}

\author{
Bernd Schäfer ${ }^{*}$, Tobias Mey ${ }^{1}$, Klaus Mann ${ }^{1}$ \\ Barbara Keitel $^{2}$, Svea Kreis ${ }^{2}$, Marion Kuhlmann², Elke Plönjes ${ }^{2}$, Kai Tiedtke ${ }^{2}$ \\ ${ }^{1}$ Laser-Laboratorium Göttingen, Hans-Adolf-Krebs-Weg 1, 37077 Göttingen, Germany \\ ${ }^{2}$ Deutsches Elektronen Synchrotron, Notkestraße 85, D-22607 Hamburg, Germany
}

\begin{abstract}
Beam parameters of the free-electron laser FLASH @13.5 nm in two different operation modes were determined from beam profile measurements and subsequent reconstruction of the Wigner distribution function behind the ellipsoidal focusing mirror at beamline BL2. 40 two-dimensional single pulse intensity distributions were recorded at each of 65 axial positions around the waist of the FEL beam with a magnifying EUV sensitized CCD camera. From these beam profile data the Wigner distribution function based on different levels of averaging could be reconstructed by an inverse Radon transform. For separable beams this yields the complete Wigner distribution, and for beams with zero twist the information is still sufficient for wavefront determination and beam propagation through stigmatic systems. The obtained results are compared to wavefront reconstructions based on the transport of intensity equation. A future setup for Wigner distribution measurements of general beams is discussed.
\end{abstract}

Keywords: wavefront, Wigner distribution, spatial coherence

\section{INTRODUCTION}

A couple of important applications of free electron lasers (FEL) require more comprehensive beam characterization than can be gained from standard techniques as beam profiling ${ }^{1}$ or caustic measurements ${ }^{2}$. In some situations even wavefront sensing techniques ${ }^{3-8}$ are not fully satisfying, in particular if the intensity and phase distributions are required at arbitrary planes were measurement is not possible and a numerical propagation of the measured field amplitude is necessary. The latter approach requires highly accurate wavefront data and a complete coherent wave field, which may be correct for seeded FEL's but surely becomes insufficient when considering self-amplified spontaneous emission (SASE)-type FEL's with inherent stochastic features ${ }^{15-17}$. On the other hand, phase space tomography i.e. the reconstruction of the Wigner distribution function ${ }^{24}$ from several profiles along the beam caustic which has already been demonstrated for X-ray synchrotron sources ${ }^{21-23}$ and recently been applied to $\mathrm{FLASH}^{9,10}$, delivers in principle a complete beam characterization including wavefront, propagation and spatial coherence ${ }^{18,19,20}$. However, unlike Hartmann and Hartmann-Shack measurements as well as coherence experiments based on Young's double slit experiment ${ }^{11-14}$, the technique gives only average results and no single pulse information. Furthermore, the Wigner-reconstructions of the FLASH beam carried out so far are based on a very limited data set obtained from standard caustic measurements, which maps only a 3D-sub-manifold of the 4D-phase space.

Following a brief introduction of the Wigner distribution we will identify those beam properties that can be estimated from standard caustic measurements and other ones which require a more complex setup. In a second section wavefront reconstructions from the Wigner function are compared to an approach using the transport of intensity equation, and finally we propose a setup for mapping almost the complete 4D-phase space.

\footnotetext{
*bernd.schaefer@llg-ev.de ; phone +49-(0)551-5035-46; fax +49-(0)551-5035-99
} 


\section{THEORY}

2.1 The Wigner distribution

The Wigner distribution $\mathrm{h}$ of a quasi-monochromatic paraxial beam is defined in terms of the mutual intensity $\mathrm{J}$ as a twodimensional Fourier transform of the latter according to ${ }^{20}$ :

$$
h(\mathbf{x}, \mathbf{u})=\left(\frac{k}{2 \pi}\right)^{2} \iint J\left(\mathbf{x}-\frac{s}{2}, \mathbf{x}+\frac{\mathbf{s}}{2}\right) e^{i k \mathbf{u s}} d^{2} s
$$

where $\mathbf{x}=(\mathrm{x}, \mathrm{y}), \mathbf{s}=\left(\mathrm{s}_{\mathrm{x}}, \mathrm{s}_{\mathrm{y}}\right)$ are two-dimensional spatial and $\mathbf{u}=(\mathrm{u}, \mathrm{v})$ angular coordinates in a plane perpendicular to the direction of beam propagation and $\mathrm{k}$ the mean wave number of light, respectively. As $\mathrm{J}$ is Hermitian, $\mathrm{h}$ is real, although it may become negative in some regions. However, its marginal distributions with respect to $\mathbf{x}$ and $\mathbf{u}$ are always non negative and yield the irradiance (near field) $\mathrm{I}(\mathbf{x})$ and the radiant intensity (far field) $\mathrm{I}_{\mathrm{FF}}\left(\mathbf{u}\right.$ ) respectively ${ }^{26}$.

The propagation of the Wigner distribution through static and lossless paraxial systems, signified by a $4 \times 4$ optical ray propagation $\mathrm{ABCD}$ matrix $\mathrm{S}$ from an input (i) to an output (o) plane writes ${ }^{20,25}$ :

$$
h_{i}(D \mathbf{x}-B \mathbf{u},-C \mathbf{x}+A \mathbf{u})=h_{o}(\mathbf{x}, \mathbf{u})
$$

Likewise, the four dimensional Fourier transform $\tilde{h}$ of h obeys a similar transformation law under propagation ${ }^{20}$ :

$$
\tilde{h}_{i}\left(A^{T} \mathbf{w}+C^{T} \mathbf{t}, B^{T} \mathbf{w}+D^{T} \mathbf{t}\right)=\tilde{h}_{o}(\mathbf{w}, \mathbf{t}),
$$

where $(\mathbf{w}, \mathbf{t})$ are the Fourier-space coordinates corresponding to $(\mathbf{x}, \mathbf{u})$.

Considering a set $\{p\}$ of parameters and a set of irradiance profiles $I_{\{p\}}(x, y)$ recorded at positions which are connected to an arbitrary reference plane via the corresponding ray transformation matrices $\mathrm{S}_{\{\mathrm{p}\}}$, one obtains, according to the marginal property of $\mathrm{h}$ and well known Fourier relations:

$$
\iint h_{\{p\}}(x, y, u, v) d^{2} u v=I_{\{p\}}(x, y) \stackrel{F T}{\leftrightarrow} \tilde{I}_{\{p\}}\left(w_{x}, w_{y}\right)=\tilde{h}_{\{p\}}\left(w_{x}, w_{y}, 0,0\right)
$$

and from (3) and (4)

$$
\tilde{h}_{r e f}\left(A_{\{p\}}^{T} \mathbf{w}, B_{\{p\}}^{T} \mathbf{w}\right)=\tilde{I}_{\{p\}}(\mathbf{w})
$$

\subsection{Reconstruction and evaluation of the Wigner distribution from standard caustic measurements}

Considering an experimental arrangement as shown in fig. 2, the parameter set $\{\mathrm{p}\}$ is simply the camera z-position and eq. (5) rewrites:

$$
\tilde{h}_{r e f}(\mathbf{w}, z \cdot \mathbf{w})=\tilde{I}_{\{z\}}(\mathbf{w})
$$

The corresponding map $\left(\mathbf{w}^{\prime}\right) \rightarrow(\mathbf{w}, \mathbf{t}): \quad\left(\begin{array}{l}w_{x} \\ w_{y} \\ t_{x} \\ t_{y}\end{array}\right)=w_{x}{ }^{\prime}\left(\begin{array}{l}1 \\ 0 \\ z \\ 0\end{array}\right)+w_{y}{ }^{\prime}\left(\begin{array}{l}0 \\ 1 \\ 0 \\ z\end{array}\right)$

can be looked upon as a 1D set of planes with set parameter z. It obeys the relation $w_{x} t_{y}-w_{y} t_{x}=0$ which is the functional equation of the 3D-submanifold $\mathrm{M}$ :

$$
M:\left\{(\mathbf{w}, \mathbf{t}) \in \mathbb{R}^{2} x \mathbb{R}^{2} \mid w_{x} t_{y}-w_{y} t_{x}=0\right\}
$$

of the reciprocal phase space covered by the standard caustic measurement ${ }^{1}$. In the following we will make use of the notation:

$$
\left.\tilde{h}\right|_{M}=\left\{\begin{array}{cc}
\tilde{h}(\mathbf{w}, \mathbf{t}) & \text { if }(\mathbf{w}, \mathbf{t}) \in M \\
0 & \text { else }
\end{array}\right.
$$

\footnotetext{
${ }^{1}$ Here we assume, the complete range of z-positions from $(-\infty, \infty)$ has either been measured or remaining gaps are filled in by interpolation.
} 
We can now ask the question which amount of information can be extracted from a Wigner distribution whose 4D Fourier transform is known merely on M. Regarding the marginal distributions it is straightforward that they can be reconstructed:

$$
I(\mathbf{x})=\iint h(\mathbf{x}, \mathbf{u}) d u d v \stackrel{F T}{\Leftrightarrow} \tilde{h}(\underbrace{\mathbf{w}, \mathbf{0}}_{\in \mathbf{M}}) \text { and } I_{F F}(\mathbf{u})=\iint h(\mathbf{x}, \mathbf{u}) d x d y \stackrel{F T}{\Leftrightarrow} \tilde{h}(\underbrace{\mathbf{0}, \mathbf{t}}_{\in \mathbb{M}^{\mathbf{0}}})
$$

From (7) it follows immediately, that the pure angular and pure spatial $2^{\text {nd }}$ moments of the Wigner distribution can be calculated as well. Considering the mixed moments, we have first:

$$
\langle u x\rangle=P^{-1} \iint u x h(\mathbf{x}, \mathbf{u}) d^{2} \mathbf{x} d^{2} \mathbf{u}=P^{-1} \frac{i}{k} \partial_{w_{x}} \partial_{t_{x}} \tilde{h}(\mathbf{w}=\mathbf{0}, \mathbf{t}=\mathbf{0})
$$

with $P$ the total beam power. The evaluation of the right hand side in the second equality of (8) requires the knowledge of $\tilde{h}$ in the $\left(\mathrm{w}_{\mathrm{x}}, \mathrm{t}_{\mathrm{x}}\right)$-plane near the origin. Therefore, as $\left(\mathrm{w}_{\mathrm{x}}, 0, \mathrm{t}_{\mathrm{x}}, 0\right) \in \mathrm{M},<\mathrm{ux}>$ can be determined and the same holds for the $<\mathrm{yv}>$ term. Obviously, the anti-diagonal parts of the mixed-moment matrix $<\mathrm{uy}>$ and $<\mathrm{vx}>$ cannot in general be calculated separately using only reciprocal phase space values on M. However, using the propagation law eq. (3) one obtains:

$$
\left.\partial_{w_{y}} \partial_{t_{x}} \tilde{h}_{z=0}\right|_{(\mathbf{0}, \mathbf{0})}+\left.\partial_{w_{x}} \partial_{t_{y}} \tilde{h}_{z=0}\right|_{(\mathbf{0}, \mathbf{0})}=\left.\partial_{w_{x}} \frac{\partial_{w_{y}} \tilde{h}_{z=d z}-\partial_{w_{y}} \widetilde{h}_{z=0}}{d z}\right|_{(\mathbf{0}, \mathbf{0})}
$$

As $\partial_{w_{x}} \partial_{w_{y}} \tilde{h}$ can be calculated whenever $(\mathbf{w}, \mathbf{0}) \in \mathrm{M}$ and $\left.\tilde{h}\right|_{M}$ can be reconstructed at arbitrary z-planes, $<$ uy $>$ and $<\mathrm{vx}>$ may be determined, provided the condition $\langle\mathrm{uy}\rangle=\langle\mathrm{vx}\rangle$ or equivalently $\left.\langle\mathrm{uy}\rangle_{-}<\mathrm{vx}\right\rangle=0$ holds. The latter equation is known as the familiar definition of the twisted phase parameter. In conclusion we may state that the second order moments of the complete 4D Wigner distribution can be determined from the $3 \mathrm{D}$ function $\left.\tilde{h}\right|_{M}$ provided that the beam under test has vanishing twist.

In a similar fashion the local moments of the beam may be treated, however, here we concentrate on the most important case of the first angular moments determining the wavefront $w$ and the transverse part $\mathbf{S}_{\perp}=\left(\mathbf{S}_{\mathbf{x}}, \mathbf{S}_{\mathbf{y}}\right)$ of the Poynting-vector S:

$$
I(\mathbf{x})^{-1} \iint\left(\begin{array}{l}
u \\
v
\end{array}\right) h(\mathbf{x}, \mathbf{u}) d u d v=I(\mathbf{x})^{-1}\left(\begin{array}{l}
S_{x} \\
S_{y}
\end{array}\right)=\left(\begin{array}{l}
\partial_{x} w \\
\partial_{y} w
\end{array}\right)+\mathbf{v}=\nabla_{\perp} w+\mathbf{v}
$$

where the source-free part $\mathbf{v}$ satisfies $\operatorname{div} \mathbf{v}=0$. By taking the Fourier transform, eq. (10) writes:

$$
\mathbf{w} \cdot \tilde{\mathbf{S}}_{\perp}=\left.\mathbf{w} \cdot \nabla_{t} \tilde{h}(\mathbf{w}, \mathbf{t})\right|_{\mathbf{t}=\mathbf{0}}=\left.\frac{\widetilde{h}_{z=d z}-\widetilde{h}_{z=0}}{d z}\right|_{\mathbf{t}=\mathbf{0}} \quad \stackrel{F T}{\leftrightarrow} \quad \nabla_{\perp} \cdot \mathbf{S}_{\perp}=\frac{I_{z=d z}-I_{z=0}}{d z}
$$

thus, from $(\mathbf{w}, \mathbf{0}) \in \mathrm{M}$ it follows, that $\mathbf{w} \cdot \tilde{\mathbf{S}}$ is attainable from $\left.\tilde{h}\right|_{M}$ and so is $\nabla_{\perp} w$. Of course, according to the divergence operation in (11), only the curl-free part of $\mathbf{S}_{\perp} / \mathbf{I}$ can be determined.

In order to figure out the possible ray transformation $\mathrm{ABCD}$-matrices, that leave the functional equation defining $\mathrm{M}$ invariant, we write the latter in symplectic form:

$$
\left(w_{x}, t_{x}\right) \cdot \mathbf{J} \cdot\left(\begin{array}{c}
w_{y} \\
t_{y}
\end{array}\right)=\left(w_{x}, t_{x}\right) \cdot\left(\begin{array}{cc}
0 & 1 \\
-1 & 0
\end{array}\right) \cdot\left(\begin{array}{c}
w_{y} \\
t_{y}
\end{array}\right)=0
$$

from which it is obvious, that $\mathrm{x}$ and $\mathrm{y}$ terms are not permitted to mix up by allowable transformations. We then get:

$$
\left(\begin{array}{cc}
A_{x} & B_{x} \\
C_{x} & D_{x}
\end{array}\right) \cdot\left(\begin{array}{cc}
0 & 1 \\
-1 & 0
\end{array}\right) \cdot\left(\begin{array}{cc}
A_{y} & C_{y} \\
B_{y} & D_{y}
\end{array}\right)=\left(\begin{array}{cc}
0 & 1 \\
-1 & 0
\end{array}\right)
$$

and therefore $A_{x}=A_{y}, B_{x}=B_{y}, C_{x}=C_{y}$ and $D_{x}=D_{y} .4 x 4$ ray matrices whose $2 \times 2$ submatrices are proportional to the $2 \times 2$ unit matrix are called stigmatic and we may state: The information contained in $\left.\tilde{h}\right|_{M}$ is sufficient for the propagation of arbitrary beams through stigmatic optical systems.

In the remainder of this section we will discuss briefly the effect of the reduced information contained in $\left.\tilde{h}\right|_{M}$ on the determination of spatial coherence. For that purpose we express the global degree of coherence $\mathrm{K}^{27}$ in terms of $\tilde{h}$ : 


$$
K=\lambda^{2} \frac{\iint h(\mathbf{x}, \mathbf{u})^{2} d^{2} \mathbf{x} d^{2} \mathbf{u}}{\left[\iint h(\mathbf{x}, \mathbf{u}) d^{2} \mathbf{x} d^{2} \mathbf{u}\right]^{2}}=\lambda^{2} \frac{\iint|\widetilde{h}(\mathbf{w}, \mathbf{t})|^{2} d^{2} \mathbf{w} d^{2} \mathbf{t}}{|\widetilde{h}(\mathbf{0}, \mathbf{0})|^{2}}
$$

which follows from Parseval's theorem.

Obviously, as M is a null set in $\mathbb{R}^{4}:\left.\quad K\right|_{M}=\lambda^{2} \frac{\left.\iint|\widetilde{h}|_{M}(\mathbf{w}, \mathbf{t})\right|^{2} d^{2} \mathbf{w} d^{2} \mathbf{t}}{|\widetilde{h}(\mathbf{0 , 0})|^{2}}=0$

However, eq. (15), although true analytically, does not hold, if the discrete space of numerical calculation with voxelsize $\delta$ is considered. In that case, points with distance $<\delta / 2$ from $M$ in each dimension will contribute to the finite sum replacing (15) and one may write:

$$
\left.K\right|_{M, \delta}=\sum_{\text {supp }_{i} \cap M \neq \varnothing}^{\text {voxelsi }_{0}}\left\langle\left.|\tilde{h}|_{M \cap s u p p_{i}}\right|^{2}\right\rangle_{i} \mathbf{V o l}_{4 D}(i)
$$

where we have normalized $\left.\tilde{h}\right|_{M}$ to $\left.\tilde{h}\right|_{M}(\mathbf{0}, \mathbf{0})=1$. Furthermore, if we introduce dimensionless variables $\mathrm{w}_{\mathrm{x}, \mathrm{y}}=\mathrm{w}_{\mathrm{x}, \mathrm{y}} / \mathrm{w}_{\max }$, $t_{x, y}=t_{x, y} / t_{\max }$, the $4 \mathrm{D}$ volume and the support of $i$ are then given by:

$$
\begin{gathered}
\operatorname{Vol}_{4 D}(i)=\delta^{4}=\frac{16}{N^{4}} \\
\operatorname{supp}_{i}=\left\{(\mathbf{w}, \mathbf{t}):\left|w_{x}-w_{x i}\right|<\frac{\delta}{2} \wedge\left|w_{y}-w_{y i}\right|<\frac{\delta}{2} \wedge\left|t_{x}-t_{x i}\right|<\frac{\delta}{2} \wedge\left|t_{y}-t_{y i}\right|<\frac{\delta}{2}\right\}
\end{gathered}
$$

with $\mathrm{N}$ the number of voxels per dimension and $\left(\mathbf{w}_{\mathbf{i}}, \mathbf{t}_{\mathbf{i}}\right)$ the center coordinates of voxel $\mathrm{i}$.

The amount eq. (16) underestimates the true global degree of coherence depends on the mapping strategy, the amount of interpolation and on the shape of $\tilde{h}$ itself. Fig. 1 shows the base-10 logarithm of the fraction

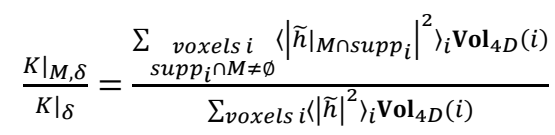

for an isotropic Gaussian Wigner distribution $\mathrm{h}$, using nearest neighbor mapping and no interpolation as a function of the width of $\tilde{h}$ in Fourier space. As expected, $\left.\mathrm{K}\right|_{\mathrm{M}, \delta} /\left.\mathrm{K}\right|_{\delta}$ decreases with higher resolutions approaching to the continuum limit. On the other hand, for $\mathrm{N}$ fixed the fraction increases with decreasing size of the Wigner distribution in Fourier space, as the $4 \mathrm{D}$ space is more densely sampled near the origin of the coordinate system than in the outer parts. In cases where the Wigner function is known to be quite isotropic, at least after an appropriate coordinate scaling, $\mathrm{K}$ may be corrected by means of eq. (17). Table 1 show $\mathrm{K}$ values with and without isotropic correction for several caustic sequences recorded at FLASH beamline BL2. The values calculated according to eq. (16) are almost a factor of ten smaller than results reported earlier which were based on recordings using a $3 \mathrm{~mm}$ aperture stop for beam size reduction ${ }^{10}$.

Table 1: Global degree of coherence K calculated from Wigner reconstruction based on standard caustic measurements for several sequences with varying number of profiles or bunch charge q, respectively. The last column shows corrected values based on the assumption of an isotropic Gaussian shaped Wigner function of 5 voxel 1/ $\mathrm{e}^{2}$-diameter.

\begin{tabular}{|l|l|l|l|l|}
\hline & No. of voxels & $\begin{array}{l}\text { No. of Profiles in re- } \\
\text { construction }\end{array}$ & K [eq. (16)] & K corrected [eq. (17)] \\
\hline Sequence 1; q=0.1 nC & $64^{4}$ & $65 @ 65$ z-Pos. & $1.5610^{-2}$ & 0.04 \\
\hline Sequence 2; q=0.1 nC & $64^{4}$ & $1300 @ 65$ z-Pos. & $9.810^{-3}$ & 0.025 \\
\hline Sequence 3; q=0.5 nC & $64^{4}$ & $65 @ 65$ z-Pos. & $5.510^{-3}$ & 0.014 \\
\hline Sequence 4; q=0.5 nC & $64^{4}$ & $1300 @ 65$ z-Pos. & $4.910^{-3}$ & 0.013 \\
\hline
\end{tabular}

However, the decrease of spatial coherence with increasing bunch charge due to the higher saturation of the FEL is quite reasonable although the corrected $\mathrm{K}$ values shown in the last column of table 1 are still considerably smaller than results obtained from Young's double pinhole experiment ${ }^{12}$. The distinguished amount of averaging as well as the incompleteness of phase space mapping may be a reason for the observed difference. 


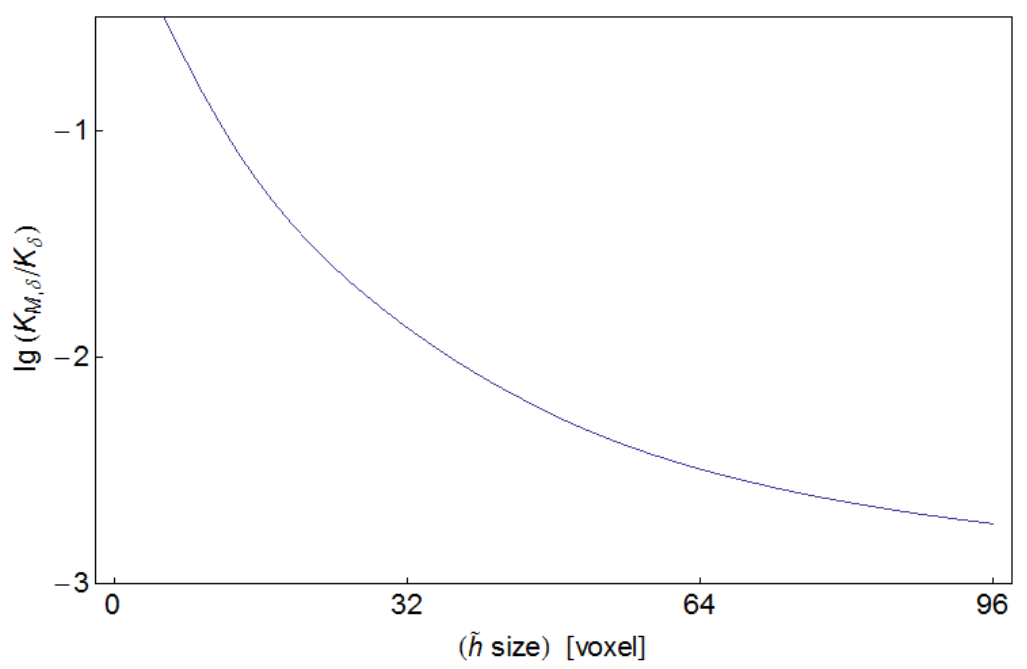

Figure 1: : Base-10 logarithm of $\mathrm{K}_{\mid \mathrm{M}, \delta} / \mathrm{K}_{\mid \delta}$ calculated according to eq. (17) for an isotropic Gaussian $\tilde{h}$-distribution as function of 1/e-distribution size in Fourier space (cf text).

\subsection{Wavefront reconstruction from the transport of intensity equation}

The conservation of energy flux in beam propagation through lossless, homogeneous and isotropic media requires:

$$
\nabla \cdot S=0
$$

where the Poynting vector $\mathbf{S}$ in a scalar theory can be written as:

$$
\mathbf{S}=\frac{1}{2 i k}\left(u^{*} \nabla u-u \nabla u^{*}\right)
$$

By writing the field amplitude $\mathrm{u}$ in terms of intensity and wavefront $: u=\sqrt{I} e^{i k w}$ it follows from (18):

$$
\nabla \cdot(I \nabla w)=0
$$

and, since $(\nabla w)_{z} \approx 1$ (paraxial approximation) one obtains the transport equation ${ }^{29}$ :

$$
\frac{\partial I}{\partial z}=-\nabla_{\perp} I \cdot \nabla_{\perp} w-I \Delta_{\perp} w
$$

$\nabla_{\perp}$ denoting the 2D Nabla operator. Eq. (21) states, that the wavefront (up to a constant term) can be determined from the irradiance distribution in adjacent planes $\mathrm{z}_{0}$ and $\mathrm{z}_{0}+\mathrm{dz}$ perpendicular to the direction of beam propagation.

For a numerical treatment $\mathrm{w}$ is written as a series expansion in terms of the Zernike polynomials $\mathrm{Z}_{1}(\mathrm{x}, \mathrm{y})^{28}$ :

$$
w(x, y)=\sum_{l=0}^{L} C_{l} Z_{l}(x, y)
$$

With (22) it follows from (21):

$$
\frac{\partial I}{\partial z}=-\sum_{l} C_{l}\left\{\frac{\partial I}{\partial x} \frac{\partial z_{l}}{\partial x}+\frac{\partial I}{\partial y} \frac{\partial z_{l}}{\partial y}+I\left(\frac{\partial^{2} z_{l}}{\partial x^{2}}+\frac{\partial^{2} z_{l}}{\partial y^{2}}\right)\right\}
$$


The discrete version of (23) at sampling points $\left(\mathrm{x}_{\mathrm{k}}, \mathrm{y}_{\mathrm{k}}\right)$ writes:

$$
\partial_{z} I_{k}+\sum_{l} C_{l}\left[\partial_{x} I_{k} \partial_{x} Z_{k, l}+\partial_{y} I_{k} \partial_{y} Z_{k, l}+I_{k}\left(\partial_{x}^{2} Z_{k, l}+\partial_{y}^{2} Z_{k, l}\right)\right]=0 ; k=1, \ldots N
$$

or

$$
B_{k}+A_{k l} C_{l}=0 \quad \mathrm{k}=1, \ldots, \mathrm{N} \text {. }
$$

After approximation of the derivatives by the familiar central differences (p: detector pixel size)

$$
\begin{aligned}
& \partial_{z} I_{k}=\frac{I\left(x_{k}, y_{k}, z_{0}+d z\right)-I\left(x_{k}, y_{k}, z_{0}\right)}{d z} \\
& \partial_{x} I_{k}=\frac{I\left(x_{k}+p, y_{k}, z_{0}+d z\right)-I\left(x_{k}-p, y_{k}, z_{0}+d z\right)}{4 p}+\frac{I\left(x_{k}, y_{k}+p, z_{0}\right)-I\left(x_{k}, y_{k}-p, z_{0}\right)}{4 p} \quad \partial_{y} I_{k}=\cdots,
\end{aligned}
$$

Eq. (24) becomes a linear NxL system with the total number of sampling points $\mathrm{N}$ and the degree of series expansion $\mathrm{L}$, that can numerically be solved by standard methods (e.g. singular value decomposition) of linear theory.

\section{EXPERIMENTAL}

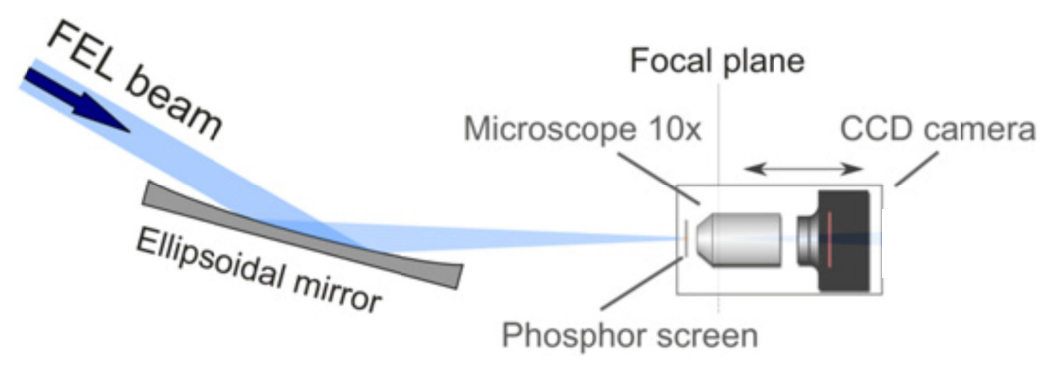

Figure 2: Experimental setup for determination of the Wigner distribution of the FLASH beam at BiL2 from standard caustic measurements (cf. text).

Figure 2 shows the setup for the FEL beam caustic measurement conducted at FLASH BL2 at $\lambda=13.5 \mathrm{~nm}$ fundamental wavelength. A carbon-coated ellipsoidal mirror with $2 \mathrm{~m}$ focal length focuses the beam down to approximately $50 \mu \mathrm{m}$ FWHM. An Al filter was used to attenuate the beam for the caustic measurement. The caustic sensor, a phosphorous screen imaged onto a 12 bit $2 / 3$ " CCD chip with $1280 \times 1024$ pixels resolution by a $10 x$ magnifying microscope on a translation stage, senses the FEL beam near the focal plane of the ellipsoidal mirror. Beam profile measurements were taken at $\mathrm{N}_{\mathrm{z}}=65 \mathrm{z}$ positions within an interval of $240 \mathrm{~mm}$ distributed around the beam waist in order to fill the phase space as uniform as possible and covering 8 Rayleigh lengths $z_{R}$ in both axial directions. At each z-position 20-40 single pulses were recorded.
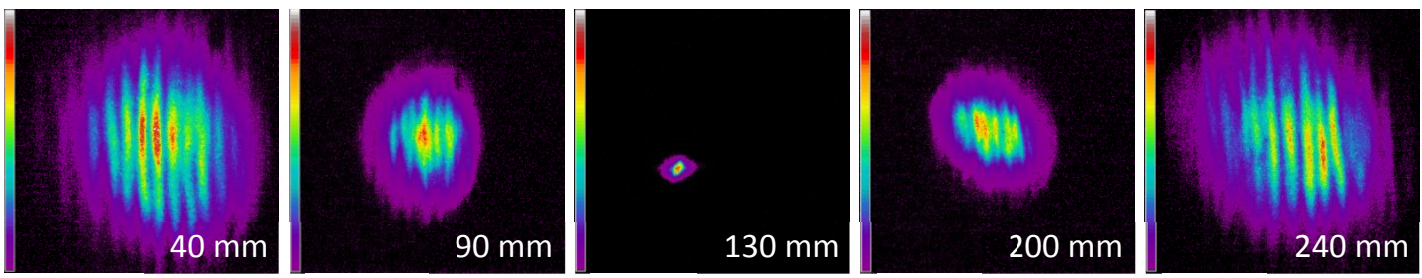

Figure 3: Single pulse beam profiles behind the elliptical mirror recorded at different z-position along the caustic of FLASH BL2. The selected area of the detector corresponds to $0.85 \times 0.66 \mathrm{~mm}^{2}$. 


\section{RESULTS}

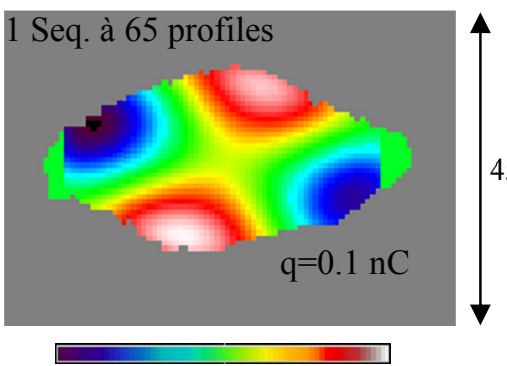

$-13 \mathrm{~nm}$

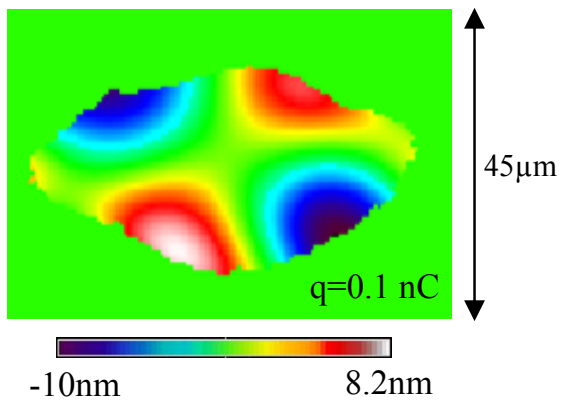

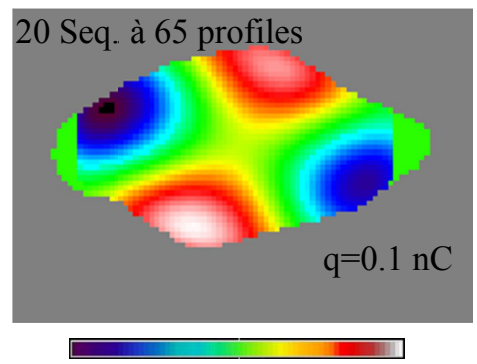

$-12 \mathrm{~nm}$

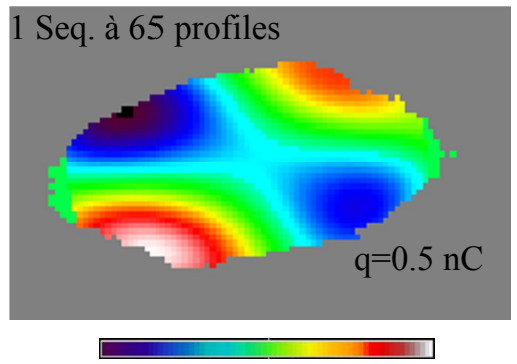

$-11 \mathrm{~nm}$

$17 \mathrm{mn}$

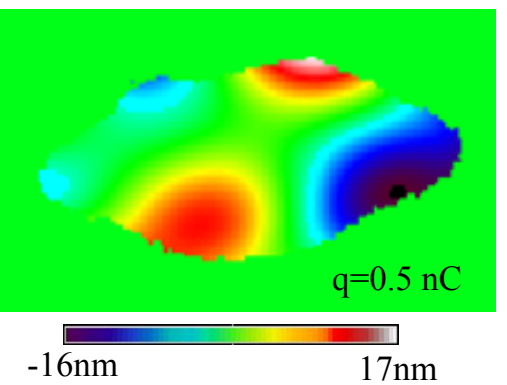

Figure 4: Wavefront at the waist of the FLASH beam at BL2 behind the elliptical mirror reconstructed from the Wigner distribution (upper row) and from the transport equation (lower row) for values of the bunch charge of $0.1 \mathrm{nC}$ and $0.5 \mathrm{nC}$, respectively. The profiles for wavefront reconstruction from the transport equation were taken at z-positions of $132 \mathrm{~mm}$ and $133 \mathrm{~mm}$ with respect to the camera reference frame.

Figure 4 shows reconstructed wavefront distributions at the waist of the FEL beam obtained from the Wigner distribution based on 1 and 20 sequences á 65 profiles recorded +-8 Rayleigh lengths around waist as well as from the transport equation using 2 single profiles near the waist position. For both methods a Zernike expansion degree of 28 polynomials has been used. The agreement is quite satisfactory, revealing the fact, that the wavefront determination requires only information localized to the vicinity of the reconstruction plane. Despite the average concordance, however, the pulse-topulse variation is considerable, showing 30\% fluctuation of the peak-valley wavefront irregularity, as shown in fig. 5 . On the other hand, the average wavefront shape near waist happens to be rather insensitive to the charge of the electron gun as can be discovered from figures 4 and 5 showing distributions for $0.1 \mathrm{nC}$ and $0.5 \mathrm{nC}$ bunch charge respectively.
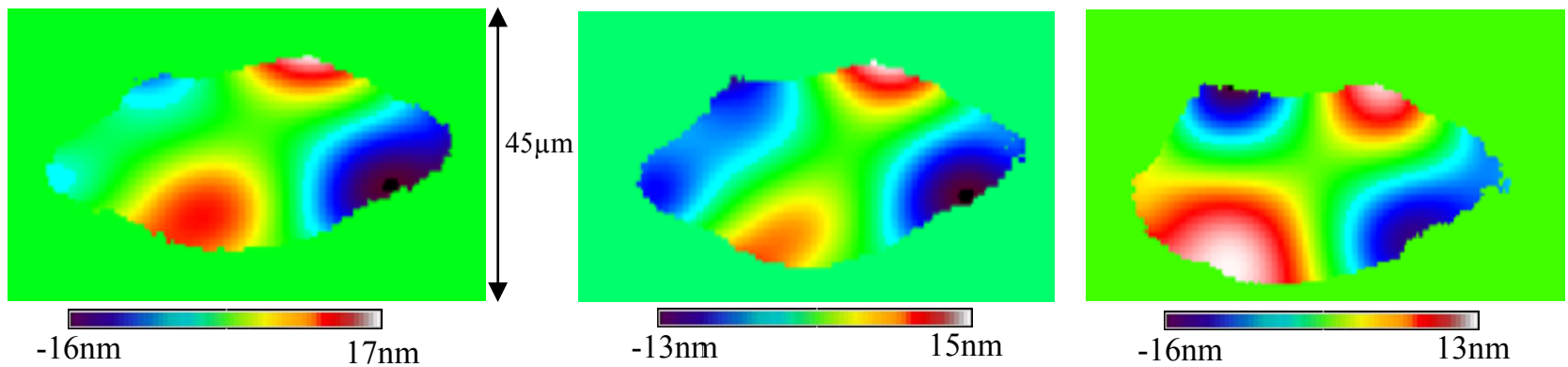

Figure 5: Pulse-to-pulse fluctuation of the wavefront near the waist of the FEL beam (BL2) @ 0.5nC bunch charge, reconstructed from the transport equation for different pulses of a sequence. Profiles from $z=132 \mathrm{~mm}$ and $133 \mathrm{~mm}$ (left and middle figure) as well as from $131.5 \mathrm{~mm}$ and $133.5 \mathrm{~mm}$ (right figure) are used for reconstruction. 
Table 2: Wavefront-RMS and peak valley values as well as number (ISO 10110-5) of the three largest selected Zernike coefficients from different evaluation methods for the FEL beam at waist position.

\begin{tabular}{|l|l|l|l|l|l|}
\hline value [nm] & & & $\begin{array}{l}\text { Zernike } \\
\text { Largest }\end{array}$ & $\begin{array}{l}\text { coefficient } \\
2^{\text {nd }} \text { largest }\end{array}$ & $3^{\text {rd }}$ largest \\
\hline $\begin{array}{l}\text { Wigner } \\
\text { 65 Profiles }\end{array}$ & 8.4 & 31.5 & $\begin{array}{l}\text { C16 } \\
\text { Fourfold }\end{array}$ & $\begin{array}{l}\text { C4 } \\
\text { Astigmatism }\end{array}$ & $\begin{array}{l}\text { C8 } \\
\text { Spherical }\end{array}$ \\
\hline $\begin{array}{l}\text { Wigner } \\
\text { 20 Seq. á 65 Profiles }\end{array}$ & 8.7 & 30 & $\begin{array}{l}\text { C16 } \\
\text { Fourfold }\end{array}$ & $\begin{array}{l}\text { C4 } \\
\text { Astigmatism }\end{array}$ & $\begin{array}{l}\text { C8 } \\
\text { Spherical }\end{array}$ \\
\hline $\begin{array}{l}\text { Intensity Transport } \\
>_{\text {over 8 single reconstructions }}\end{array}$ & 4.3 & 25 & $\begin{array}{l}\text { C16 } \\
\text { Fourfold }\end{array}$ & $\begin{array}{l}\text { C4 } \\
\text { Astigmatism }\end{array}$ & $\begin{array}{l}\text { C3 } \\
\text { Defocus }\end{array}$ \\
\hline
\end{tabular}

Despite the broader database, the Wigner-based wavefront reconstruction delivers more distinct distributions than the transport-of-intensity approach when compared in terms of the wavefront RMS-deformation and, less pronounced, in the peak-valley data. The pulse-to-pulse fluctuations give rise to a considerable randomness in data taken from only two profiles. In turn, as the random part spreads evenly over the whole Zernike-spectrum and happens to be uncorrelated to the wavefront signal, the amplitude of the latter, located at lower spatial frequencies is reduced to some extent. Table 2 gives an overview for the wavefront RMS- and peak-valley-deformation as well as the number of the three largest Zernike coefficients according to ISO 10110-5 @ 0.1nC bunch charge.

\section{SETUP FOR SAMPLING THE COMPLETE 4D PHASE SPACE}

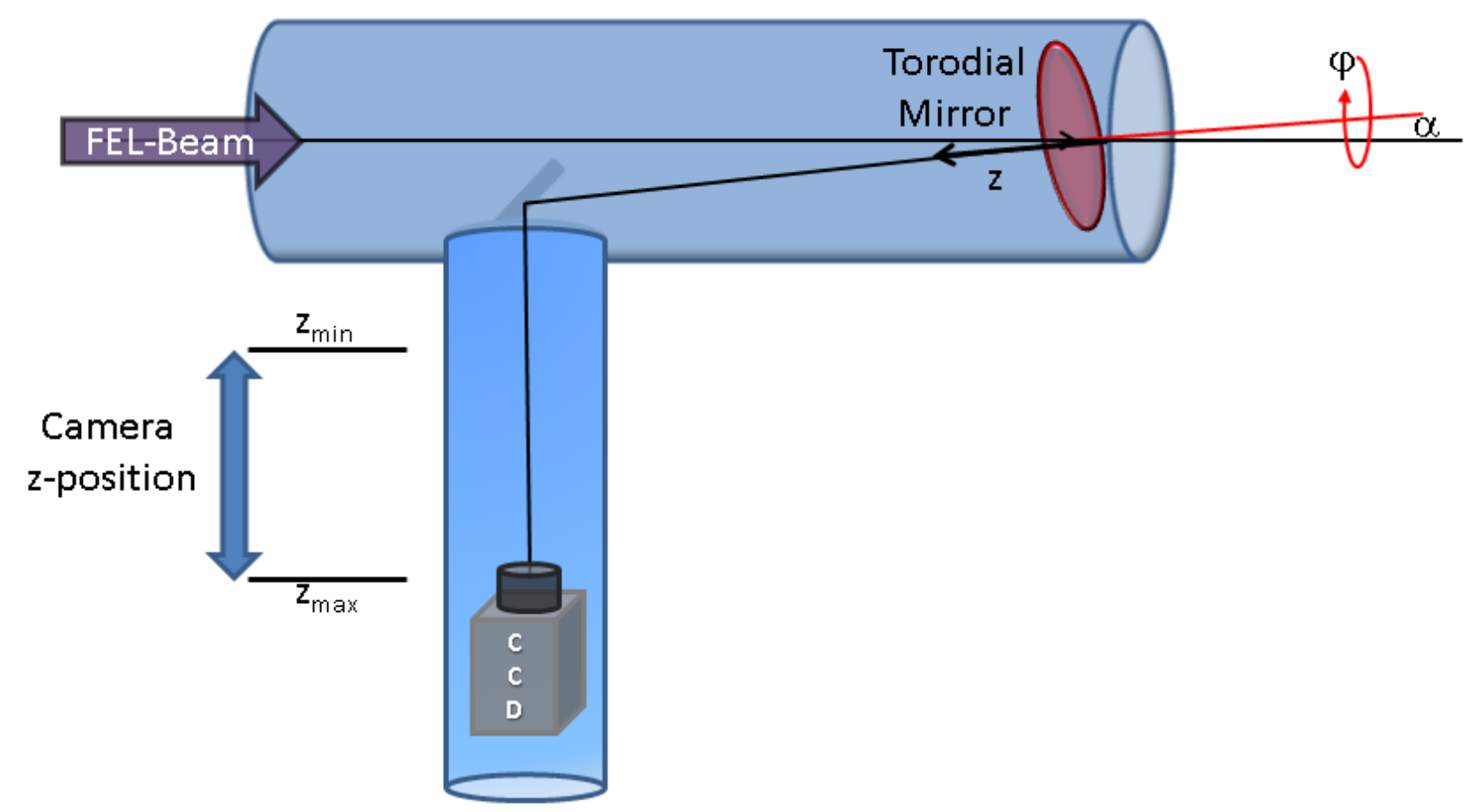

Figure 6: Experimental setup for measuring a 4D Wigner function.

Figure 6 shows a principal setup, that enables the measurement of a 4D section of phase space. Compared to the caustic setup shown in fig. 2, the grazing incidence ellipsoidal mirror is replaced by a toroidal mirror, whose surface normal is tilted by a small angle $\alpha$ with respect to the incoming beam. Furthermore, in addition to the camera z-movement the toroid can be rotated around its normal by an angle $\varphi \in[0,2 \pi]$. Both parameters $\mathrm{z}$ and $\varphi$ together with the detector plane 
coordinates $\mathrm{x}$ and $\mathrm{y}$, respectively, constitute a parameter space, being sufficient for 4D phase space mapping. Without loss of generality let the reference plane be a fixed distance $\mathrm{z}$ ' in front of the toroid mirror. Then the $4 \times 4$ transformation matrix $\mathrm{S}_{\{p\}}=\mathrm{S}_{\{\varphi, z\}}$ from the reference plane to the camera position writes:

$$
S_{\{\varphi, z\}}=S_{z} S_{\text {tilt }}{ }^{-1} R_{\varphi}^{-1} S_{\text {Toroid }} R_{\varphi} S_{\text {tilt }} S_{Z^{\prime}}
$$

The matrices $\mathrm{S}_{\mathrm{z}}, \mathrm{S}_{\mathrm{z}}$, and $\mathrm{S}_{\text {Toroid }}$ denote free space propagation and the focussing action of the toroid according to :

$$
S_{z}=\left(\begin{array}{cccc}
1 & 0 & z & 0 \\
0 & 1 & 0 & z \\
0 & 0 & 1 & 0 \\
0 & 0 & 0 & 1
\end{array}\right) \quad \text { and } \quad S_{\text {Toroid }}=\left(\begin{array}{cccc}
1 & 0 & 0 & 0 \\
0 & 1 & 0 & 0 \\
-2 / R_{x} & 0 & 1 & 0 \\
0 & -2 / R_{y} & 0 & 1
\end{array}\right)
$$

whereas $\mathrm{R}_{\varphi}$ and $\mathrm{S}_{\text {tilt }}$ symbolize rotation and tilt of the toroid mirror. They are given by:

$S_{\text {tilt }}=\left(\begin{array}{cccc}\sqrt{\cos \alpha} & \multicolumn{1}{c}{0} & 0 & 0 \\ 0 & \sqrt{\cos \alpha}^{-1} & 0 & 0 \\ 0 & 0 & \sqrt{\cos \alpha}^{-1} & 0 \\ 0 & 0 & 0 & \sqrt{\cos \alpha}\end{array}\right)$ and $R_{\varphi}=\left(\begin{array}{cccc}\cos \varphi & \sin \varphi & 0 & 0 \\ -\sin \varphi & \cos \varphi & 0 & 0 \\ 0 & 0 & \cos \varphi & \sin \varphi \\ 0 & 0 & -\sin \varphi & \cos \varphi\end{array}\right)$, respectively.

The resulting 4D-map explicitly writes:

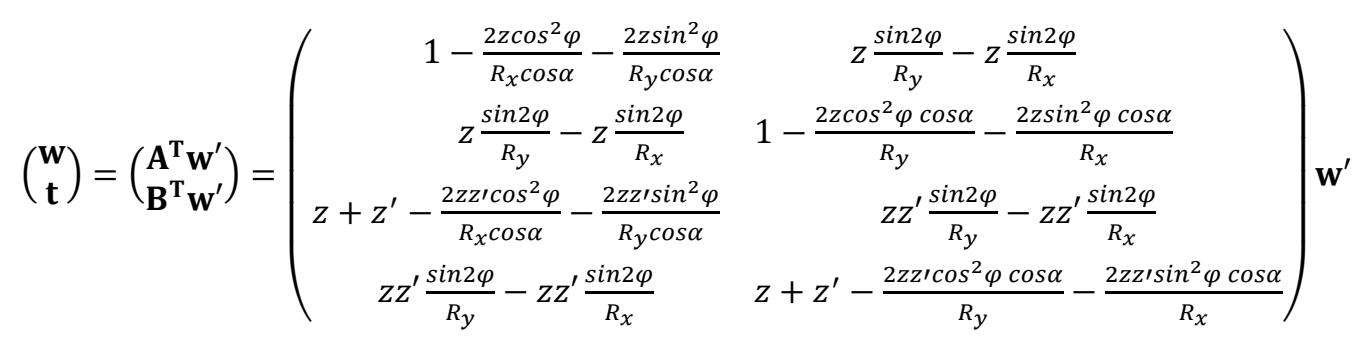

The proof, that eq. (26) covers the complete phase space, provided $\left\{\mathrm{w}_{\mathrm{x}}{ }_{\mathrm{x}}, \mathrm{w}_{\mathrm{y}}, \varphi, \mathrm{z}\right\}$ passes through its whole data range, is straightforward, however, the calculations are quite lengthy, and we omit it here for reasons of space. The interested reader is referred to ${ }^{25}$, where the analysis is performed for a similar setup containing a spherical and a rotating cylindrical lens.

\section{CONCLUSION}

In the preceding it has been shown, that the Wigner reconstruction based on a standard caustic measurement gives already valuable information of beam parameters and wavefront. Moreover, if one is interested only in stigmatic optical systems, the propagation of such an incompletely sampled beam can thoroughly be performed. In the case of complete spatial coherence, or if solely the wavefront distribution is of concern, however, it has been shown in sects. 3 and 4, that an analysis based on an even further reduced data set of only two neighbouring irradiance profiles can successfully be done by using the transport of intensity equation. Wavefronts in the waist of FLASH @ beamline BL2 have consistently been reconstructed from both techniques.

On the other hand, the results presented in section 3 clearly show, that the measurement of spatial coherence, the complete characterization of beam carrying a twisted phase or beam propagation through arbitrary systems cannot be performed based on such a reduced data set. Only in the very special case of a separable beam complete beam information is available from the incomplete sampled Wigner distribution. Although early experimental results supported the separability of FLASH BL2, this does obviously no longer hold in the absence of limiting apertures. In those cases a proper characterisation of partially coherent beams requires a full 4D extension of the standard caustic approach which is proposed and briefly discussed in section 5. In particular, when coherence properties of the beam are of major importance, the wide variety of information gained may outweigh the higher experimental effort. 


\section{ACKNOWLEDGEMENT}

This work was supported by Deutsche Forschungsgemeinschaft within SFB755 'Nanoscale Photonic Imaging. Support of the FLASH user facility through the BMBF program FSP301-FLASH is greatly acknowledged. We also acknowledge support from the German federal ministry of economics and technology within the ZIM joint project WKALAS.

\section{REFERENCES}

[1] ISO 11146-1,2,3, Lasers and laser-related equipment - Test methods for laser beam widths, divergence angles and beam propagation ratios, International Organization for Standardization (ISO), Geneve (2004/2005)

[2] ISO/DIS 13694, Lasers and laser related equipment - Test methods for laser beam power (energy) distribution, International Organization for Standardization (ISO), Geneve (1998)

[3] Flöter, B. et al, "Beam parameters of FLASH beamline BL1 from Hartmann wavefront measurements", Nucl. Instr. and Meth. A (2010), doi:10.1016/j.nima.2010.10.016

[4] Flöter, B., Juranic, P., Kapitzki, S., Keitel, B., Mann, K., Plönjes, E., Schäfer, B., Tiedtke, K., "EUV Hartmann sensor for wavefront measurements at free-electron laser FLASH", New J. Phys. 12, 083015 (2010)

[5] Kuhlmann, M. et al, "Wave-front observations at FLASH", Proc. FEL 2006 (Berlin: BESSY) (2006)

[6] Mercère, P. et al, "Hartmann wave-front measurement at $13.4 \mathrm{~nm}$ with accuracy", Opt. Lett 28 (2003)

[7] Schäfer, B. and Mann, K., "Determination of beam parameters and coherence properties of laser radiation by use of an extended Hartmann-Shack wave-front sensor", Appl. Opt 41 (2002)

[8] Neal, D.R., Alford, W.J. ,Gruetzner, J.K., Warren, M.E., "Amplitude and phase beam characterization using a twodimensional wavefront sensor", Proc. SPIE 2870 72-82 (1996)

[9] Schäfer, B., Flöter, B., Mann, K., Plönjes, E., Keitel, B., Tiedtke, K., "Beam characterization of FLASH from Hartmann data and measurement of the Wigner distribution function", Proc. SPIE 8078-19, (2011)

[10] Schäfer, B., Flöter, B., Mey, T., Juranic, P., Kapitzki, S., Plönjes, E., Keitel, B., Mann, K., Tiedtke, K., "FEL beam characterization from measurement of the Wigner distribution function", Nucl. Instr. And Meth. A (2011), doi:10.1016/j.nima.2011.07.031

[11] Mitzner, R. et al. , "Spatio-temporal coherence of free electron laser pulses in the soft x-ray regime", Opt. Exp. 16, 19909 (2008)

[12] Singer, A., Vartanyants, I.A. ,Kuhlmann, M. , Duesterer, S., Treusch, R., Feldhaus, J., ”Transverse-Coherence Properties of the Free-Electron-Laser FLASH at DESY”, PRL 101, 254801 (2008)

[13] Mitzner, R. , Rolling, S., Siemer, B., Wöstmann, M., Tiedtke, K., Feldhaus, J., Zacharias, H., "Direct evaluation of the temporal and spatial coherence properties of FLASH pulses", DESY annual report (2009)

[14] Ischebeck, R. et al., "Study of the Transverse Coherence at the TTF Free Electron Laser", Nucl. Instrum. Meth. A 507, 175-180 (2003)

[15] Krinsky, S., Li, Y., "Statistical analysis of the chaotic optical field from a self amplified spontaneous emission free electron laser", Phys. Rev. E 73, 066501 (2006).

[16] Saldin, E.L., Schneidmiller, E.A., Yurkov, M.V., "Diffraction effects in the self-amplified spontaneous emission FEL", Optics Communications 186, 185-209 (2000)

[17] Saldin, E.L., Schneidmiller, E.A., Yurkov, M.V., "Statistical and coherence properties of radiation from x-ray freeelectron lasers", New J. Phys. 12, 035010 (2010)

[18] L. Mandel and E. Wolf, [Optical Coherence and Quantum optics], Cambridge University Press, $1^{\text {st }}$ ed., Cambridge (1995)

[19] Eppich, B., Mann, G., Weber, H., "Measurement of the four dimensional Wigner distribution of paraxial light sources", in Proc. SPIE 5962-2, D1-D11 (2005)

[20] McAlister, D.F., Beck, M., Clarke, L., Mayer, A., Rayner, M.G., "Optical phase retrieval by phase space tomography and fractional-order Fourier transforms", Opt. Lett., 20, 1181-1183 (1995)

[21] Cámara, A., Alieva, T. ,Rodrigo, J.A., Calvo, M.L., "Tomographic Reconstruction of the Wigner Distribution of Non-separable Beams", PIERS Cambridge 2010 Proc. ISSN 1559-9450 (2010) 
[22] Tran, C.Q., Peele, A.G., Roberts, A., Nugent, K.A., Paterson, D. and McNulty, I., ”X-ray imaging: a generalized approach using phase-space tomography". J. Opt. Soc. Am. A-Optics Image Science and Vision, 22(8), 1691-1700 (2005)

[23] Tran, C.Q., Williams, G.J., Roberts, A., Flewett, S., Peele, A.G., Paterson, D., de Jonge, M.D., Nugent, K.A., "Experimental measurement of the four-dimensional coherence function for an undulator x-ray source", Phys. Rev. Lett., 98(22), 224801 (2007)

[24] Bastiaans, M.J., "Application of the Wigner distribution function to partially coherent light", J. Opt. Soc. Am. A, 3(8), (1986)

[25] Schäfer, B., Mann, K., "Characterization of an ArF excimer laser beam from measurements of the Wigner distribution function", New J. Phys., 13043013 (2011)

[26] Bastiaans, M.J., "The Wigner distribution function for partially coherent light", Optica acta (28) 9, (1981)

[27] Eppich, B., "Definition, meaning and measurement of coherence parameters", Proc. SPIE 4270, pp. 71-79, (2001)

[28] M. Born and E. Wolf, [Principles of Optics], Cambridge University Press, $7^{\text {th }}$ ed., Cambridge (2001)

[29]D. Malacara, ed. [Optical Shop Testing], pp. 425, John Wiley \& Sons, Inc., $3^{\text {rd }}$ ed., Hoboken (2007) 